\title{
DNA methylation mediates the effect of maternal smoking on offspring birthweight: a birth cohort study of multi-ethnic US mother- newborn pairs
}

\author{
Richard Xu', Xiumei Hong ${ }^{2 *} \mathbb{0}$, Boyang Zhang ${ }^{3}$, Wanyu Huang ${ }^{4}$, Wenpin Hou ${ }^{3}$, Guoying Wang ${ }^{2}$,
} Xiaobin Wang ${ }^{2,5}$, Tak Igusa ${ }^{4}$, Liming Liang ${ }^{6,7}$ and Hongkai $\mathrm{Ji}^{3}$

\begin{abstract}
Background: Maternal smoking affects more than half a million pregnancies each year in the US and is known to result in fetal growth restriction as measured by lower birthweight and its associated long-term consequences. Maternal smoking also has been linked to altered fetal DNA methylation (DNAm). However, what remains largely unexplored is whether these DNAm alterations are merely markers of smoking exposure or if they also have implications for health outcomes. This study tested the hypothesis that fetal DNAm mediates the effect of maternal smoking on newborn birthweight.
\end{abstract}

Methods: This study included mother-newborn pairs from a US predominantly urban, low-income multi-ethnic birth cohort. DNAm in cord blood were determined using the Illumina Infinium MethylationEPIC BeadChip. After standard quality control and normalization procedures, an epigenome-wide association study (EWAS) of maternal smoking was performed using linear regression models, controlling for maternal age, education, race, parity, prepregnancy body mass index, alcohol consumption, gestational age, maternal pregestational/gestational diabetes, child sex, cord blood cell compositions and batch effects. To quantify the degree to which cord DNAm mediates the smoking-birthweight association, the VanderWeele-Vansteelandt approach for single mediator and structural equational model for multiple mediators were used, adjusting for pertinent covariates.

Results: The study included 954 mother-newborn pairs. Among mothers, 165 (17.3\%) ever smoked before or during pregnancy. Newborns with smoking exposure had on average $258 \mathrm{~g}$ lower birthweight than newborns without exposure $(P<0.001)$. Using a false discovery rate $(F D R)<0.05$ as the significance cutoff, the EWAS identified 38 differentially methylated $\mathrm{CpG}$ sites associated with maternal smoking. Of those, $17 \mathrm{CpG}$ sites were mapped to previously reported genes: GFI1, AHRR, CYP1A1, and CNTNAP2; 8 of those, located in the first three genes, were Bonferroni significantly associated with newborn birthweight and mediated the smoking-birthweight association. The combined mediation effect of the three genes explained $67.8 \%$ of the smoking-birthweight association.

Conclusions: Our study not only lends further support that maternal smoking alters fetal DNAm in a multiethnic population, but also suggests that fetal DNAm substantially mediates the maternal smoking-birthweight association.

\footnotetext{
*Correspondence: xhong3@jhu.edu

${ }^{2}$ Center On the Early Life Origins of Disease, Department of Population,

Family and Reproductive Health, Johns Hopkins University Bloomberg School of Public Health, Baltimore, MD, USA

Full list of author information is available at the end of the article
}

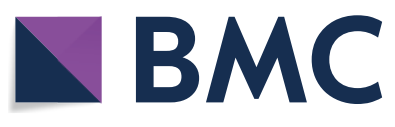

(c) The Author(s) 2021. Open Access This article is licensed under a Creative Commons Attribution 4.0 International License, which permits use, sharing, adaptation, distribution and reproduction in any medium or format, as long as you give appropriate credit to the original author(s) and the source, provide a link to the Creative Commons licence, and indicate if changes were made. The images or other third party material in this article are included in the article's Creative Commons licence, unless indicated otherwise in a credit line to the material. If material is not included in the article's Creative Commons licence and your intended use is not permitted by statutory regulation or exceeds the permitted use, you will need to obtain permission directly from the copyright holder. To view a copy of this licence, visit http://creativecommons.org/licenses/by/4.0/. The Creative Commons Public Domain Dedication waiver (http://creativeco mmons.org/publicdomain/zero/1.0/) applies to the data made available in this article, unless otherwise stated in a credit line to the data. 
Our findings, if further validated, indicate that DNAm modification is likely an important pathway by which maternal smoking impairs fetal growth and, perhaps, even long-term health outcomes.

Keywords: Maternal smoking, Low birthweight, DNA methylation, Epigenome-wide association study, Mediation analysis

\section{Background}

In the US, about $12 \%$ of pregnant women continue to smoke during pregnancy [1], which translates to more than half a million smoking-affected pregnancies each year [2]. Maternal smoking during pregnancy has been known to adversely affect fetal growth, as reflected in lower birthweight compared to the non-smoking group [3]. More recently, maternal smoking also has been linked to altered fetal DNA methylation (DNAm); these findings are consistent based on either self-reported smoking or objective measurement of blood cotinine levels [4-6]. The importance of these observations reaches far beyond the perinatal period. Low birthweight has been linked to a range of pediatric disorders [7] and chronic diseases later in life, including obesity, diabetes, cardiovascular diseases, and stroke, as highlighted by the Barker Hypothesis [8]. Such a link between low birthweight and chronic diseases was later confirmed by numerous studies around the world [9]. In recent years, prospective birth cohort studies [10, 11] have lent further support for the concept of developmental origins of health and disease (DoHaD) [12, 13].

Despite mounting evidence of $\mathrm{DoHaD}$, the underlying biological mechanisms driving them remain to be understood. One plausible hypothesis points to epigenetic modification, which regulates gene expression without changing the original DNA sequence. Specifically, the epigenome operates at the interface between the genome and the environment; is largely established in-utero; and is particularly sensitive to early life environmental perturbation [14]. DNAm is a well-studied epigenetic mechanism, and its stability over decades makes it a very useful biomarker to study early life environmental exposures and life-course health [14]. DNAm in cord blood at multiple sites have also been found to be significantly associated with birthweight, as supported by an increasing number of studies [1518 ] including the meta-analysis in the Pregnancy And Childhood Epigenetics Consortium [16]. Some of birthweight-related $\mathrm{CpG}$ sites overlap with those reported to be associated with maternal smoking [16]. In this context, maternal smoking, fetal DNAm and birthweight assessed together could serve as an exemplifying model to gain deeper insight into $\mathrm{DoHaD}$.
This study aimed to fill in research gaps and to advance the field in the following ways. First, there is strong evidence that maternal smoking can affect fetal DNAm as measured in cord blood, and a list of related genes are consistently found across studies $[4,5,19]$. To date, all the related published studies were based on use of the Illumina HumanMethylation27 or 450 BeadChip, while a higher density BeadChip, with higher coverage of methylation dynamic regions, such as the enhancers, is now available. More importantly, a significant gap is the lingering lack of data for disadvantaged populations, especially US urban, low-income African American populations, who are at high-risk of low birthweight and chronic diseases. Second, despite robust findings for the association between maternal smoking and fetal DNAm, it remains to be determined whether the smoking-associated DNAm alterations merely represent markers of smoking exposure or also are biomarkers of smokingassociated adverse health effects. Mediation analyses could help to address this question by linking maternal smoking (exposure), DNAm (mediator) and birthweight (health outcome) together and dissecting the direct effects of smoking and indirect (mediation) effects of smoking via DNAm. To our knowledge, a limited number of studies of this kind were conducted in European samples using the 450 BeadChip, [20, 21] while none have been conducted with African Americans.

This study was designed to analyze data gathered from mother-newborn pairs enrolled in the Boston Birth Cohort, a predominantly urban, low-income multiethnic (Blacks and Hispanics) sample in Boston, MA. It sought to accomplish the following objectives: first, to conduct an epigenome-wide association study (EWAS) to identify novel genes and replicate known genes with altered methylation levels associated with maternal smoking during pregnancy, using the latest Illumina Infinium MethylationEPIC BeadChip. Second, to examine whether smoking-associated DNAm alterations are also associated with newborn birthweight. Third, to estimate to what degree smoking-associated methylation alterations in cord blood individually and collectively mediate the smoking-birthweight association. To our knowledge, this is the first large study of this kind in a high-risk US multiethnic population, with application of advanced mediation analyses to quantify 
individual $\mathrm{CpG}$ sites and their combined mediation of the smoking-birthweight association.

\section{Results}

\section{Population characteristics}

After data quality control steps (see "Methods" section), the current study included a total of 954 mothernewborn dyads; of those, 165 (17.3\%) newborns were exposed to maternal ever smoking during pregnancy and 789 were unexposed. Compared with unexposed newborns, exposed newborns had 258 g lower birthweight (2911 vs. $3169 \mathrm{~g}, P<0.001$, Table 1). Compared to non-smokers, smoking mothers had higher prepregnancy BMI $(P=0.007)$, higher rates of alcohol consumption $(P<0.001)$, and lower education level $(P<0.001)$. Gestational age was slightly shorter in exposed newborns compared to non-exposed (38.3 vs. 38.7 weeks, $P=0.058)$. Other factors including maternal age at delivery, parity, maternal pregestational/gestational diabetes and newborn sex were comparable between the two groups (all $P>0.05$, Table 1).

\section{EWAS with maternal smoking in cord blood}

Figure 1 shows the Manhattan plot and QuantileQuantile plot of the association between maternal smoking and cord blood DNAm. At a false discovery rate $(\mathrm{FDR})<0.05$ as the genome-wide significance cutoff, we identified $38 \mathrm{CpG}$ sites with altered DNAm in newborns exposed to maternal smoking compared to unexposed newborns. Of those, $17 \mathrm{CpG}$ sites were mapped to four previously reported genes: growth factor independent 1 transcriptional repressor (GFI1), Aryl-hydrocarbon receptors repressor (AHRR), cytochrome P450, family 1, member A1 (CYP1A1), and contactin associated protein 2 (CNTNAP2). Additional file 1: Table 1 presents details on the $38 \mathrm{CpG}$ sites in terms of their chromosomal positions, corresponding gene names, their location within a gene, coefficients of DNAm association with smoking using inverse-normal transformed beta values, nominal $P$ values, and FDR. The CpG sites in the GFI1, AHRR and CNTNAP2 genes were significantly hypomethylated as indicated by the negative coefficients, and CpG sites in the $C Y P 1 A 1$ gene were hypermethylated as indicated by the positive coefficients in relation to maternal smoking. We then explored the inter-correlations among the $38 \mathrm{CpG}$ sites associated with maternal smoking via heatmap, as shown in Fig. 2. We found that CpG sites in the GFI1 and CYP1A1 genes were highly correlated. We used factor analysis to create a composite score to represent highly correlated $\mathrm{CpG}$ sites in the GFI1 and CYP1A1 genes (named as GFI1 gene score and CYP1A1 gene

Table 1 Characteristics of 954 mother-newborn pairs from the Boston Birth Cohort by maternal smoking status during pregnancy

\begin{tabular}{|c|c|c|c|}
\hline & No smoking & Smoking & $P^{a}$ \\
\hline N & 789 & 165 & \\
\hline \multicolumn{4}{|l|}{ Continuous variables [mean (SD)] } \\
\hline Maternal age at delivery (years) & $28.4(6.6)$ & $27.9(6.1)$ & 0.329 \\
\hline Maternal pre-pregnancy BMI (kg/m²) & $26.7(6.2)$ & $28.1(7.0)$ & 0.007 \\
\hline Gestational age at delivery (weeks) & $38.7(2.4)$ & $38.3(2.7)$ & 0.058 \\
\hline Child birthweight (g) & $3169.1(656.1)$ & $2910.5(680.6)$ & $<0.001$ \\
\hline \multicolumn{4}{|l|}{ Categorical variables, $n(\%)$} \\
\hline Parity ( $\geq 1$ live birth) & $437(55.4)$ & $92(55.8)$ & 0.999 \\
\hline Maternal alcohol consumption & $45(5.7)$ & $34(20.6)$ & $<0.001$ \\
\hline Maternal education level (> high school) & $286(36.2)$ & $33(20.0)$ & $<0.001$ \\
\hline Maternal race & & & 0.457 \\
\hline Black & $566(71.7)$ & $113(68.5)$ & \\
\hline Non-Black & $223(28.3)$ & $52(31.5)$ & \\
\hline Maternal diabetes & & & 0.333 \\
\hline No & $700(88.7)$ & $141(85.5)$ & \\
\hline Gestational diabetes & $51(6.5)$ & $16(9.7)$ & \\
\hline Pregestational diabetes & $38(4.8)$ & $8(4.8)$ & \\
\hline Child sex (male) & $410(52.0)$ & $94(57.0)$ & 0.278 \\
\hline
\end{tabular}

$S D$ standard deviation, $B M /$ body mass index

a Population characteristics was compared between those mothers who ever smoked during pregnancy versus those who did not, using the chi-square test for categorical variables and ANOVA for continuous variables 

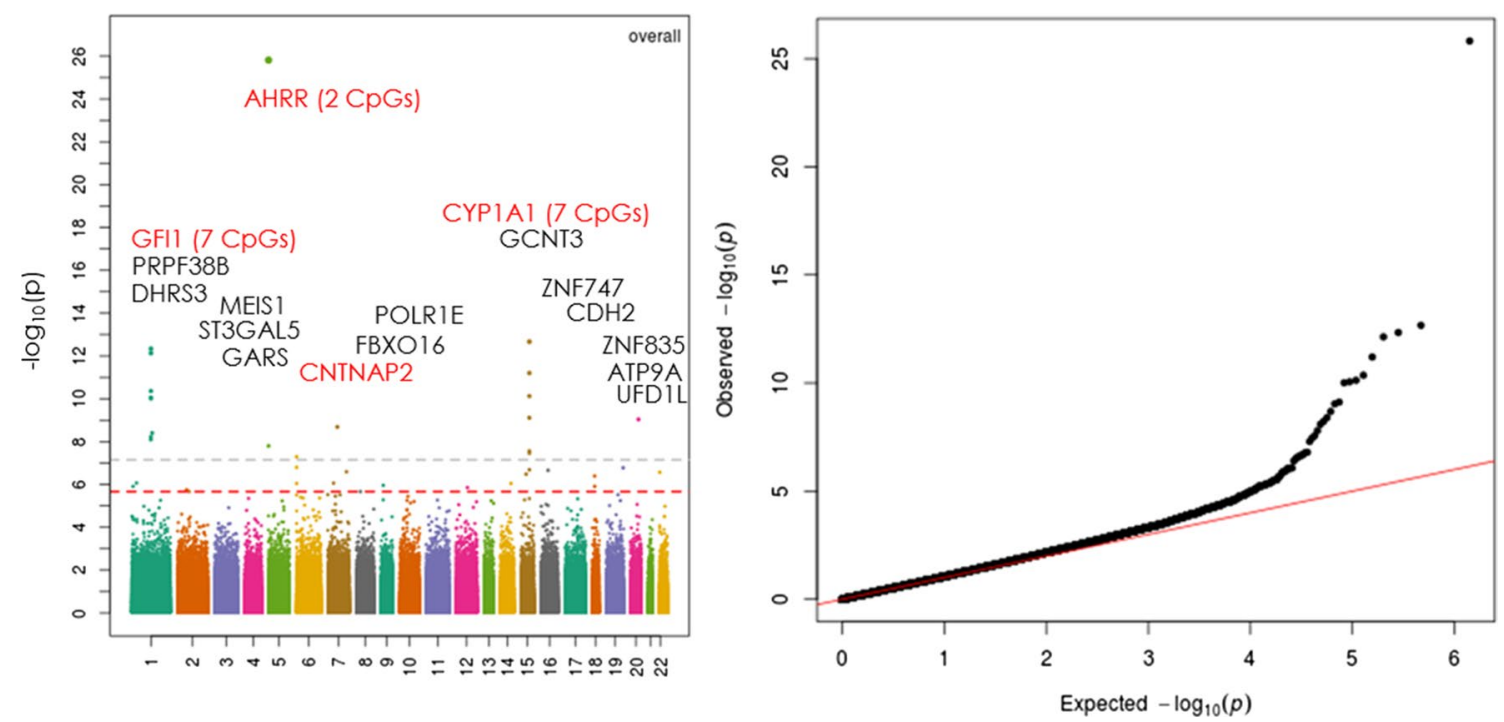

Fig. 1 Manhattan plot of the association between maternal smoking during pregnancy and cord blood DNA methylation (left panel); and Quantile-Quantile plot of the association between maternal smoking during pregnancy and cord blood DNA methylation (right panel), in 954 mother-newborn pairs in the Boston Birth Cohort

score, respectively), which were applied in the next step's gene-based mediation analysis.

As a sensitivity analysis, when maternal smoking was analyzed as an ordered three-categorical variable $(0=$ never, $1=$ quitting during pregnancy; $2=$ continuous smoking, Additional file 1: Fig. 1), we noticed that there was an inverse and dose-response relation between maternal smoking and DNAm as represented by the GFI1 gene score (Panel A) and DNAm of cg05575921 in the AHRR gene (Panel B), with the lowest DNAm among newborns exposed to continuous smoking; and the same pattern was observed for newborn birthweight (Panel D). In contrast, there was a positive association between maternal smoking categories and DNAm as represented by the CYP1A1 gene score (Panel C), with the highest DNAm among newborns exposed to continuous smoking. Given the limited sample size, our subsequent analyses were limited to the binary smoking categories: never vs. ever smoking. We have further performed EWAS with maternal smoking, stratified by maternal race (Blacks vs nonBlacks) (Additional file 1: Fig. 2; Additional file 1: Table 2). Of note, the main EWAS results for the overall sample appeared to be driven by Blacks. We found that a CgG site (cg05549655) in the CYP1A1 gene was significantly associated with maternal smoking in both the Black and non-Black group. Similarly, CpGs sites in the other two known genes (GFI1, AHRR) also tended to have comparable associations with maternal smoking in both groups, although the associations in non-Blacks were no longer significant after FDR adjustment. There were also some CpGs sites which had associations with maternal smoking in Blacks only, as summarized in Additional file 1: Table 2 . We have also tested smoking $\times$ race interactions on epigenome-wide DNAm, and we found only 1 significant interaction with CpG site cg14650464 (the FANCC gene), but this CpG site showed no association with birthweight (data not shown).

\section{Mediation effects of cord blood DNAm on the smoking- birthweight relationship}

As illustrated in Additional file 1: Fig. 3, our data met the prerequisite for mediation analyses. Newborns with in-utero exposure to maternal smoking had $258 \mathrm{~g}$ lower birthweight than newborns without exposure $(P<0.001)$. Table 2 presents the associations between the identified 38 smoking-associated CpG sites and birthweight, with adjustment of gestational age and other covariates in the models. We found that a total of $8 \mathrm{CpG}$ sites, in the GFI1, AHRR, and CYP1A1 genes, had significant associations with birthweight at $P<0.0013$ (Bonferroni correction for 38 tests). We also performed statistical tests for DNAm $\times$ sex and DNAm $\times$ race (Black vs non-Black) interactions on birthweight, respectively, but did not detect any significant interactions (results not shown).

We then used the VanderWeele-Vansteelandt approach to estimate the mediation effect of each single CpG on the maternal smoking-newborn birthweight association. Additional file 1: Table 3 presents the mediation results for all $38 \mathrm{CpG}$ sites associated with smoking. The 


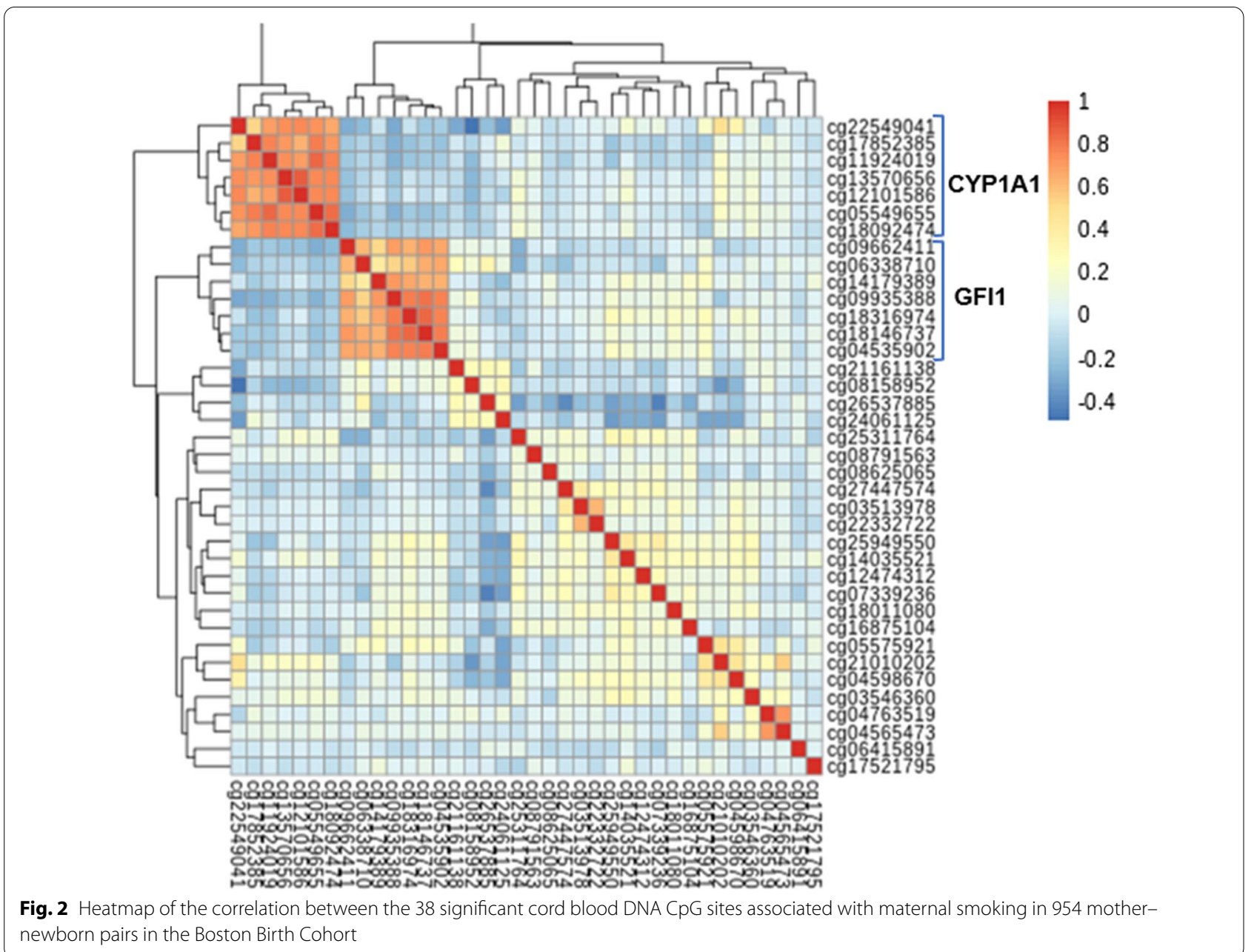

mediation effect of individual $\mathrm{CpG}$ sites ranged from 0.5 to $44.6 \%$ of the total smoking-birthweight association. Of note, aside from the CpG sites in the GFI1, AHRR and CYP1A1 genes, none of the rest CpG sites were both significantly associated with birthweight and had significant mediation effect. Therefore, the subsequent combined mediation analysis was limited to the GFI1, AHRR, and CYP1A1 genes. When the GFI1 gene score and CYP1A1 gene score were analyzed instead of single CpG site, the mediation percentage was $44.6 \%$ for the GFI1 gene and $29.8 \%$ for the CYP1A1 gene, respectively (Table 3). The combined mediation effect of the three genes (GFI1 gene score, AHRR cg05575921, CYP1A1 gene score), estimated via the structural equation model, explained $67.8 \%$ of the total smoking effect on birthweight (Table 3).

\section{Subgroup analyses}

As shown in Additional file 1: Tables 4-7, we performed sex- and race-specific subgroup mediation analyses and found that the percentage mediation by the combination of three genes (GFI1, AHRR, CYP1A1) was $79.8 \%$ in males $(n=504) ; 52.3 \%$ in females $(n=450), 79.6 \%$ in Blacks $(n=679)$, and $46.1 \%$ in non-Blacks $(n=275)$. We also tested DNAm $\times$ sex and DNAm $\times$ race (black vs non-black) interactions on birthweight, respectively. However, we did not detect any significant DNAm $\times$ sex or DNAm $\times$ race interactions (results not shown).

\section{Discussion}

Our study has made following contributions to the scientific literature with regards to in-utero exposure to maternal smoking, epigenetic modification, and offspring birthweight. This study extends previous work, all based on the Illumina Infinium Methylation27 or 450 BeadChip, $[5,6,20]$ which were biased toward promoter regions and missed more dynamic methylation sites including the enhancers. To our knowledge, this is by far the largest EWAS using the latest Illumina Infinium MethylationEPIC BeadChip (with over $850 \mathrm{~K}$ CpG sites) among a population of predominantly urban, low-income, Black 
Table 2 Association of newborn birthweight with 38 CpG sites and gene methylation scores that were significantly associated with maternal smoking during pregnancy in 954 mother-newborn pairs from the Boston Birth Cohort

\begin{tabular}{|c|c|c|c|c|}
\hline \multirow[t]{2}{*}{ Gene } & \multirow[t]{2}{*}{$\mathrm{CpG} *$} & \multicolumn{3}{|c|}{$\begin{array}{l}\text { Associations } \\
\text { with birthweight }^{\mathrm{a}}\end{array}$} \\
\hline & & Beta & SE & $P$ \\
\hline \multirow[t]{8}{*}{ GFI1 } & cg09662411 & 62.6 & 15.1 & $3.66 \mathrm{E}-05^{\#}$ \\
\hline & cg06338710 & 36.5 & 16.1 & 0.020 \\
\hline & $\operatorname{cg} 18146737$ & 65.5 & 14.9 & $1.29 E-05^{\#}$ \\
\hline & cg18316974 & 60.4 & 15.0 & $6.37 \mathrm{E}-05^{\#}$ \\
\hline & cg04535902 & 66 & 16.1 & $4.72 \mathrm{E}-05^{\#}$ \\
\hline & cg09935388 & 68.6 & 15.5 & $1.09 \mathrm{E}-05^{\#}$ \\
\hline & $\operatorname{cg} 14179389$ & 48.7 & 15.5 & 0.002 \\
\hline & GFl1 gene score & 71.5 & 15.6 & $5.17 \mathrm{E}-06^{\#}$ \\
\hline \multirow[t]{2}{*}{ AHRR } & cg05575921 & 92.0 & 15.4 & $3.00 E-09^{\#}$ \\
\hline & $\operatorname{cg} 21161138$ & 23.4 & 15.6 & 0.135 \\
\hline \multirow[t]{8}{*}{ CYPIA1 } & cg05549655 & -59.1 & 17.6 & $7.97 \mathrm{E}-04^{\#}$ \\
\hline & cg17852385 & -36.7 & 17.8 & 0.039 \\
\hline & cg13570656 & -50.4 & 16.7 & 0.003 \\
\hline & $\operatorname{cg} 12101586$ & -47 & 16.1 & 0.004 \\
\hline & cg22549041 & -32.9 & 16.1 & 0.041 \\
\hline & $\operatorname{cg} 11924019$ & -46.6 & 17.9 & 0.010 \\
\hline & $\operatorname{cg} 18092474$ & -56.5 & 17.2 & $0.001^{\#}$ \\
\hline & CYP1A1 gene score & -61.3 & 18.0 & $6.84 \mathrm{E}-04^{\#}$ \\
\hline DHRS3 & cg03546360 & 24.6 & 16.3 & 0.155 \\
\hline PRPF38B & $\operatorname{cg} 18011080$ & 21.5 & 15.6 & 0.169 \\
\hline MEIS1 & cg08791563 & 23.1 & 15.6 & 0.140 \\
\hline ST3GAL5 & cg26537885 & 2.54 & 17.8 & 0.886 \\
\hline GARS & cg16875104 & 17.8 & 16.3 & 0.274 \\
\hline CNTNAP2 & cg25949550 & 6.2 & 15.7 & 0.694 \\
\hline FBXO16 & $\operatorname{cg} 12474312$ & -3.8 & 15.6 & 0.808 \\
\hline POLRIE & cg14035521 & 47.4 & 15.4 & 0.002 \\
\hline GCNT3 & cg25311764 & -41.2 & 15.5 & 0.008 \\
\hline ZNF747 & cg17521795 & 16.3 & 15.4 & 0.291 \\
\hline \multirow[t]{2}{*}{$\mathrm{CDH} 2$} & cg03513978 & 42.8 & 15.2 & 0.005 \\
\hline & cg22332722 & 22.4 & 15.4 & 0.146 \\
\hline ZNF835 & cg08158952 & 20.5 & 14.9 & 0.169 \\
\hline ATP9A & cg07339236 & 31.3 & 17.0 & 0.065 \\
\hline UFD1L & cg08625065 & 10.4 & 16.2 & 0.518 \\
\hline \multirow[t]{7}{*}{ Intergenic CpGs } & cg06415891 & -29.4 & 16.0 & 0.067 \\
\hline & cg21010202 & 14.7 & 15.5 & 0.343 \\
\hline & cg04763519 & -4.3 & 15.8 & 0.786 \\
\hline & cg04565473 & 5.31 & 15.5 & 0.731 \\
\hline & cg04598670 & 21.5 & 15.3 & 0.160 \\
\hline & cg27447574 & 3.3 & 16.3 & 0.843 \\
\hline & cg24061125 & -19.7 & 14.9 & 0.187 \\
\hline
\end{tabular}

a Adjusted for maternal age, education level, race, parity, pre-pregnancy BMI, alcohol consumption, gestational age at delivery, pre-gestational/gestational diabetes, child sex, cord blood cell compositions (CD8, CD4, NK, B cell, monocytes, granulocytes, nucleated red blood cells)

* For each $\mathrm{CpG}$, inverse-normal transformed $\beta$-value was analyzed
Table 2 (continued)

\# Statistically significant based on Bonferroni correction $(0.05 / 38=0.0013)$

mother-newborn pairs in the US. We replicated CpG sites/genes (GFI1, AHRR, CYP1A1, and CNTNAP2) that were previously found to be associated with maternal cigarette smoking, mostly in European or non-Black populations $[4,5]$, suggesting shared associations by different ethnic population. As shown in Additional file 1: Table 1, we also identified additional significant genes, including DHRS3 (cg03546360), PRPF38B (cg18011080), MEIS1 (cg08791563), ST3GAL5 (cg26537885), GARS (cg16875104), FBXO16 (cg12474312), POLR1E (cg14035521), GCNT3 (cg25311764), ZNFZ47 (cg17521795), CDH2 (cg03513978, cg22332722), ZNF835 (cg08158952), ATP9A (cg07339236), and UFD1L (cg08625065). However, except for GFI1, AHRR, and $C Y P 1 A 1$, none of the other genes stood out as having an FDR significant association with newborn birthweight. Although we cannot dismiss their importance in DoHad, they may act through different mechanisms (rather than altered fetal growth) on health and disease later in life.

For the first time, we quantitatively estimated the degree to which smoking-related alterations in cord blood DNAm CpG sites individually and collectively mediate the smoking-birthweight association. We achieved this by applying advanced mediation analyses, including the VanderWeele-Vansteelandt method for a single mediator and structural equation model for multiple mediators. Under the VanderWeele-Vansteelandt model, we found that $8 \mathrm{CpG}$ sites in the three known genes (GFI1, $A H R R$, and CYP1A1) were significantly associated with newborn birthweight and substantially mediated the smoking-birthweight association. Under the structural equation model, the combined mediation effect of the GFI1, AHRR, and CYP1A1 genes explained $67.8 \%$ of the smoking-birthweight association. There were considerable variations in the degree of mediation across the subgroups, with the mediation effect appears to be stronger in males $(79.8 \%)$ than in females $(52.3 \%)$, and stronger in Blacks (79.6\%) than in non-Blacks (46.1\%). If our mediation results can be further confirmed, it would imply that modification of DNAm is likely a very important biological pathway by which maternal smoking impairs fetal growth and, perhaps, long-term health.

Although we cannot interpret the significant mediation as a causal relationship, our findings are robust and biologically plausible. Consistent with previous literature, we have found strong and consistent evidence that maternal smoking during pregnancy can significantly alter fetal DNAm. As shown in Additional file 1: Fig. 1, there was a dose-response relationship between fetal 
Table 3 Mediation effect of single CPG, single gene score, and combined mediation effect of the GFI1, AHRR, and CYP1A1 genes on the maternal smoking-newborn birthweight association in 954 mother-newborn pairs from the Boston Birth Cohort

\begin{tabular}{|c|c|c|c|c|}
\hline \multirow[t]{2}{*}{ Gene/CpG } & \multicolumn{4}{|c|}{ Mediated effect (VanderWeele-Vansteelandt Approach) } \\
\hline & Beta & SE & $P$ & $\%$ Mediated \\
\hline GFl1 gene score & -70.1 & 24.0 & 0.004 & 44.6 \\
\hline AHRR CpG cg05575921 & -61.7 & 28.3 & 0.029 & 38.5 \\
\hline CYPIAl gene score* & -47.9 & 15.5 & 0.002 & 29.8 \\
\hline \multirow[t]{2}{*}{ Model parameters } & & \multicolumn{3}{|c|}{$\begin{array}{l}\text { Mediation effect by structural equation model (simultaneous } \\
\text { consideration of the } 3 \text { genes) }\end{array}$} \\
\hline & & Beta & SE & $P$ \\
\hline Direct effect of maternal smoking & & -50.4 & 42.8 & 0.239 \\
\hline Indirect effect via GFI1 gene score & & -31.3 & 11.6 & 0.007 \\
\hline Indirect effect via AHRR CpG cg05575921 & & -55.2 & 13.3 & $<0.001$ \\
\hline Indirect effect via CYP1A1 gene score & & -18.6 & 8.5 & 0.022 \\
\hline Sum of indirect effect (or mediation effect) & & -106.0 & 18.3 & $<0.001$ \\
\hline Total effect of maternal smoking & & -156.4 & 40.7 & $<0.001$ \\
\hline Percent mediated & & $67.8 \%$ & & \\
\hline
\end{tabular}

All models adjusted for maternal age, education level, race, parity, pre-pregnancy BMI, alcohol consumption, pre-gestational/gestational diabetes, gestational age, child sex, cord blood cell compositions (CD8, CD4, NK, B cell, monocytes, granulocytes, nucleated red blood cells)

CpG methylation levels and maternal smoking categories (never, quitter, and continuous smoking). We have replicated CpG sites in genes that have important biological functions [4-6, 19]. For example, GFI1 is involved in diverse developmental processes, $A H R R$ plays a key role in the aryl hydrocarbon receptor signaling pathway, and $C Y P 1 A 1$ is involved in phase I metabolism, which regulates the detoxification of the components of tobacco smoke. In the future, by integrating genotyping data in mothers and infants, we will have the potential to test the causal relationship between maternal smoking and cord DNAm, and between cord DNAm and birthweight, via two-step mendelian randomization approaches [22].

Prior to this current study, there was only a limited number of studies on cord blood DNAm mediation of maternal smoking and newborn birthweight, and all of those were conducted in European populations. An early study [20] focused on 129 Dutch children exposed to maternal smoking versus 126 unexposed to maternal and paternal smoking, using the Illumina $450 \mathrm{~K}$ array and traditional regression models. It showed a significant mediation effect of DNAm in the GFI1 and NEUROG1 genes on the smoking-birthweight relationship. A more recent study [21] in 282 German children, using the Illumina $450 \mathrm{~K}$ array, also showed evidence of cord blood DNAm in the PIM1, CNTNAP2, and ITGB7 genes mediating the smoking-birthweight association, while no mediation effects were reported for DNAm in the AHRR, GFI1 and $C Y P 1 A 1$ genes. Using different sources of bio-samples, a recent study in a European sample $(n=441)$ [23] examined placental DNAm in the $M D S 2, P B X 1, C Y P 1 A 2$, $V P R B P, W B P 1 L, C D 28$, and $C D K 6$ genes as mediators of maternal smoking on birthweight and demonstrated significant mediation effects. A more recent study in a Danish case-control study of autism examined neonatal DNAm (blood sample obtained about 1 week after birth) and again found that maternal smoking was significantly associated with both birthweight and neonatal DNAm, including CpG sites in GFI1 and AHRR genes [24]

In comparison, our study, in a larger birth cohort composed of predominantly Black mother-infant pairs, confirmed DNAm mediation effects of CpG sites in the $A H R R$, GFI1 and CYP1A1 genes, and indicated that such effects tended to be stronger in Blacks. Of note, the main EWAS findings with maternal smoking in the overall sample appear to be driven by the black sample. Our tests for DNAm $\times$ race interactions on birthweight were not significant. It is unclear whether there are true differences between the Black and non-Black due to sample size constraint, especially in the non-Black subset. Our stratified analyses may be underpowered, and the findings remain to be confirmed in future studies and their implications remain to be understood. It is known that DNAm profiles are sex-specific as early as at birth. However, few studies have performed sex-stratified mediation analyses, which may be partly due to a limited sample size in previous studies. The study by Witt et al. indicated that the CpG site in CNTNAP2 had a more pronounced 
effect in male newborns. Consistently, our results showed that the DNAm mediation effects on smoking-birthweight relationships were stronger in males, suggesting that such mediation effect may be sex-dependent. However, our interaction tests for DNAm $\times$ sex were not significant. Future studies should further examine potential sex and racial differences in the DNAm and birthweight associations.

Our findings, especially the significant mediation by cord blood DNAm, if further confirmed, have important implications for research and clinical and public health practice. Beyond this study, the interplay of maternal smoking, fetal DNAm and birthweight could serve as a prototype to understand the impact of other early life environmental exposures on the developing fetus and their underlying biological mechanisms. Prospective birth cohort studies with long-term postnatal follow-up will offer important opportunities to further investigate the possibility that altered fetal DNAm may have a longlasting impact on the exposed child's health over the life course.

Our study has following limitations. First, it lacks a replication sample, and DNAm profiles were not validated using other independent technologies. However, we replicated CpG sites in the GFI1, AHRR, CYP1A1, and CNTNAP2 genes which are well-known to be associated with cigarette smoking $[4-6,19]$. We further demonstrated that the altered $\mathrm{CpG}$ sites in the first three genes were associated with newborn birthweight and significantly mediated the maternal smoking-newborn birthweight association, supporting the biological plausibility of our findings. Second, maternal smoking in this study is self-reported and may be subject to reporting bias. We validated this variable by measuring known metabolites of nicotine in a subset $(n=630)$, including cotinine and hydroxy-cotinine, in both maternal plasma and cord blood plasma (direct evidence of fetal exposure). As shown in Additional file 1: Fig. 4, there are distinct differences in the distributions of these metabolites by maternal self-reported smoking during pregnancy, and their levels were the highest in mothers and newborns with self-reported continuous smoking during pregnancy. However, there are observed overlaps in the distribution between selfreported never smoking vs. ever smoking which could be due to several reasons: (1) maternal smoking is under reported; (2) Plasma cotinine and hydroxy-cotinine can only reflect recent maternal smoking (half-life 1-3 days), which may explain partly that some smoking mothers had extremely low cotinine levels, which is possible if they did not smoke during or after delivery; and (3) exposure to passive smoking, which may partly explain why some never-smoking mothers have relatively high levels of cotinine. Although both selfreported smoking and biomarkers have limitations, to a large degree, our data lent support that self-reported smoking in our study is overall a reliable indicator of maternal smoking status. Third, despite being relatively large, our study may still be underpowered to identify CpG sites with smaller effect sizes. We also were unable to further explore observed differences in the percentage of mediation by DNAm between males and females, between Black and non-Black groups, and between quitters and continuous smokers due to limited sample sizes. Fourth, this study was conducted in a US predominantly urban, low-income, multiethnic population, which is both a strength (understudied population) and a weakness (less generalizable). The current study sample is a subset of the Boston Birth Cohort. As shown in Additional file 1: Table 8, overall, the included and excluded samples were comparable for the baseline demographic and clinical variables, except that the included sample of the current study had a higher percentage of Black participants. We are uncertain whether the higher percentage of mediation effect in Blacks was due to a larger sample size of Black participants compared to non-Black participants or if there was a real racial difference. Nevertheless, our overall findings on the effect of maternal smoking on birthweight and cord blood DNAm are remarkably consistent with previous studies across diverse populations, [4-6, 19] lending support that the smoking effect on fetal growth and DNAm is likely universal. Fifth, with increasing availability of high-dimensional epigenomic data, there is growing interest to quantify the extent to which the effect of environmental factors on health is mediated by multiple epigenetic marks or on a genome-wide scale. As underscored by a recent commentary [25], "given the lack of an overarching validated framework and the generally complex causal structure of high-dimensional data, the analysis of high-dimension mediation currently requires great caution and effort to incorporate a priori biological knowledge", more studies are needed to confirm our findings related to the degree of mediation and whether it is causal although our mediation analysis followed a priori biology and hypotheses. Finally, given the epigenome is at the interface of gene and environment, future studies are needed to add the genome to the puzzle and to further delineate the complex interplay of genome, epigenome, and environmental effects on health outcomes [26]. 


\section{Conclusion}

In this predominantly US urban, low-income multiethnic sample, using the latest Illumina Infinium MethylationEPIC BeadChip, we identified a total of 38 differentially methylated CpG sites with genome-wide significance $(\mathrm{FDR}<0.05)$ associated with maternal smoking. Of those, 8 CpG sites in the GFI1, AHRR, and CYP1A1 genes were Bonferroni significantly associated with newborn birthweight and their combined mediation effect explained $67.8 \%$ of the smoking-birthweight association. Our findings raise the possibility that altered fetal DNAm may not only serve as a biomarker of in-utero smoking exposure (quitter or continuous), but also may represent an important biological pathway by which maternal smoking impairs fetal growth, and, perhaps, even long-term health outcomes. Beyond this current study, the methodologies we used may serve as a prototype for future investigations of other in-utero adverse environmental exposures on the developing fetus and health across the life course.

\section{Methods}

\section{Study population}

Additional file 1: Fig. 5 presents the flow chart of the study participants. This study included 954 mothernewborn pairs from the Boston Birth Cohort (BBC), a US predominantly urban low-income, Black population. The BBC was initiated in 1998 with rolling enrollment at the Boston Medical Center, in Boston, MA, as detailed elsewhere [3]. In brief, mothers who delivered singleton live births at the Boston Medical Center were invited to participate shortly after giving birth. The BBC is enriched by preterm ( $<37$ weeks of gestation) and low birthweight $(<2500 \mathrm{~g})$ births due to by design over-sampling at enrollment. Pregnancies that were a result of in vitro fertilization, multiple gestations (e.g., twins, triplets), fetal chromosomal abnormalities or major birth defects or preterm birth due to trauma were excluded. After mothers gave written informed consent, they were enrolled and asked to complete a standard questionnaire interview on maternal socio-demographic characteristics, lifestyle including smoking and alcohol consumption, diet, and reproductive and medical history. Maternal and newborn clinical information including birth outcomes were obtained from their medical records. The study protocol has received initial and annual continuation approval by the Institutional Review Boards of Boston Medical Center and the Johns Hopkins Bloomberg School of Public Health. Additional file 1: Table 8 presents characteristics of the 954 pairs enrolled in this study compared to the rest mother-newborn pairs $(n=7555)$ in the BBC.

\section{Definition of maternal smoking, the primary exposure}

Maternal smoking during pregnancy was defined based on maternal questionnaire interview at enrollment: '(1) in the six months before you found out you were pregnant, did you smoke/use tobacco?'; (2) 'Did you smoke/ use tobacco in the first three months of pregnancy?; (3) 'Did you smoke/use tobacco in the middle three months of pregnancy?'; and (4) 'Did you smoke/use tobacco in the last three months of pregnancy?'. We defined 'maternal smoking during pregnancy' as those mothers who answered 'yes' to any of the above questions and defined 'non-smoking' mothers as those mothers who answered 'no' to all the above questions. Additional sensitivity analyses were performed to further divide maternal smoking into "Quitters-who stopped smoking since the 1st or 2nd or 3rd trimester" versus "Continuous smokerswho continued to smoke during the entire pregnancy". In a subset $(n=630)$, we validated self-reported maternal smoking by measuring known metabolites of nicotine, including cotinine and hydroxy cotinine, in both maternal plasma and cord blood plasma (direct evidence of fetal exposure), as shown in the Additional file 1: Fig. 4.

\section{Definition of important covariates}

Other important covariates that were adjusted in the analysis included: maternal age at delivery, parity (not including the index pregnancy, 0 versus 1 or more), maternal education (high school or less versus some college or more), maternal self-reported race (Black versus non-Black): Black included self-reported Black (African American and Haitian) and non-Black included white and Hispanic, maternal alcohol consumption during pregnancy (never versus ever), maternal pre-pregnancy body mass index (BMI), maternal pregestational/gestational diabetes (yes versus no), child's sex (female versus male), and gestational age (GEAA) at birth. As detailed in our earlier publication[3] in the BBC, GEAA was estimated using an established algorithm based on both last menstrual period (LMP) and the result of early ultrasound (U/S, <20 weeks of gestation) to maximize accuracy and minimize missing data. In this cohort, about $70 \%$ of the mothers had early $\mathrm{U} / \mathrm{S}$ and corresponding percentage of early U/S among never vs. ever smoking mothers were $72.1 \%$ vs. $68.5 \%(P=0.398)$. Most of the covariates have been previously defined and published [27].

\section{DNAm profiling in cord blood and quality control steps}

Cord blood was obtained by the trained nursing staff of the labor and delivery service; the quality of the DNA samples has been demonstrated in our previous studies using the Illumina BeadChip [27]. A total of 963 cord DNA samples (plus 21 replicates) were sent to the 
University of Minnesota Genomics Center for genomewide DNAm profiling, using the Illumina Infinium MethylationEPIC BeadChip (850K). With this platform, DNAm profiles for a total of 865,859 CpG sites (at locations of cytosines followed by guanine) were successfully generated and a $\beta$-value for each $\mathrm{CpG}$ site examined was reported, ranging from $0-1.0$, to signify the percentage of DNAm at each CpG site. Systematic quality control steps were performed using existing analytic pipelines with $\mathrm{R} /$ Bioconductor package 'minfi' [28]. Briefly, with a raw intensity file (.idat) for each sample, first, the $850 \mathrm{~K}$ control probes were examined to assess bisulfite conversion, extension, hybridization, staining, specificity and other factors. Second, the median for both Meth and Unmeth signals for each array were computed and displayed in a scatter plot to identify outlier samples with low intensity. Using a median $\log _{2}$ intensity value $<9$ as the cutoff, 1 sample that appeared to be an outlier was removed from subsequent analyses. A sensitivity analysis, performed after removing an additional 12 samples with median $\log 2$ intensity $<10$, yielded very similar results. Third, correlations for the duplicates were computed among the 21 pairs of duplicates. Fourth, although this report did not include maternal DNAm, for DNA methylation profiling, both maternal samples ( $n \sim 420$ samples) and cord DNA samples were randomly placed in each 96-well DNA plate. Lab personnel were blinded to the sample placement. Data QC steps were performed in cord samples, along with mother-child dyad and newborn sex information. Furthermore, multi-dimensional scaling (MDS) plots were used to evaluate outlier samples and confirm male cord blood samples, female cord blood samples and maternal blood samples, which clustered separately, as expected. Seven samples whose DNAm data-derived sex values were inconsistent with sex as documented in medical records were identified and removed from the subsequent analyses. Fifth, sample-wise missing rates were calculated and one sample with $>2 \%$ missing rate was removed. At the locus level, $4193 \mathrm{CpG}$ sites that had a detection $P$ value $>0.01$ in more than $5 \%$ of the samples were removed. An additional 140,271 CpG sites were removed due to the following reasons: having an annotated SNP at the measured or extension site or that overlapped with the probe, and/or that potentially crosshybridized to other genomic locations [29]. Then, we applied the single-sample Noob (ssNoob) methods via "preprocessnoob" function for background and dye bias correction [30], and then performed quantile normalization in "preprocessQuantile" to normalize type 1 and type II probes. We additionally removed $16,843 \mathrm{CpG}$ sites in the sex chromosomes. These filters resulted in high quality DNAm data for 704,552 CpG sites in 954 samples for subsequent analyses.

\section{Statistical methods}

Summary statistics were performed to compare the demographic and clinical characteristics of newborns who were exposed to maternal smoking during pregnancy versus those who were not exposed, using the chi-square test for categorical variables and ANOVA for continuous variables. We applied the following analytical methods to address the study objectives. All statistical analyses were performed using $\mathrm{R}$ (version 4.0.2; $\mathrm{R}$ Foundation for Statistical Computing).

\section{Identification of differentially methylated sites}

We investigated differentially methylated sites in cord blood that were associated with maternal smoking, using the 'limma' package [31]. To address the skewed distribution and potential outliers or bimodal distribution of the original $\beta$-values, we applied an inverse normal transformation and obtained inversely normalized $\beta$-values for each CpG. To account for potential batch effects, models were additionally adjusted with calculated surrogate variables (SV) using the 'SmartSVA' package [32]. A total of $62 \mathrm{SV}$ variables were generated that were then adjusted in the subsequent analyses. We fitted a linear regression model with the inversely-normalized $\beta$-value at each CpG site as the outcome and maternal smoking $(1=$ ever, $0=$ never) as the independent variable, adjusting for potential confounders including maternal age, education level, race, parity, pre-pregnancy BMI, alcohol consumption, gestational age, pregestational/gestational diabetes, child sex, and SV variables. We also adjusted for estimated cord blood cell composition, which was calculated using the estimateCellCounts() function in the 'minfi' package, where the distribution of each cell type, including $\mathrm{CD} 4+, \mathrm{CD} 8+\mathrm{T}$ cells, $\mathrm{B}$ cells, monocytes, granulocytes, natural killer cells, and nucleated red blood cells (specific to cord blood), was inferred for each cord blood sample, based on external cord blood reference DNA methylation signatures of the constituent cell type from the Illumina Infinium Methylation450 BeadChip [33]. The false discovery rate (FDR) [34] was applied to correct for multiple testing, with FDR $<0.05$ as the genome-wide significance cutoff. Stratified analyses by maternal race were also performed.

\section{Mediation analyses}

Additional file 1: Fig. 3 illustrates our sequential analytical methods to dissect the interplay of maternal smoking, cord blood DNAm, and newborn birthweight. As a first step, we examined whether the significant smoking-associated $\mathrm{CpG}$ sites, as identified above, are associated with newborn birthweight adjusting for GEAA, maternal age, education level, race, parity, pre-pregnancy BMI, alcohol consumption, pre-gestational/gestational 
diabetes, child sex, cord blood cell compositions; thus, we focused on their effect on fetal growth. Scatter plots of birthweight versus each $\mathrm{CpG}$ methylation site were first inspected, which supports the use of linear regression models to assess the adjusted associations between each $\mathrm{CpG}$ (as the exposure) and newborn birthweight (as the outcome). Then, for those CpG sites that were associated with maternal smoking, we estimated the degree to which maternal smoking-associated $\mathrm{CpG}$ alterations, individually and collectively, mediate the maternal smoking-birthweight association. First, we applied the VanderWeele-Vansteelandt method[35] for assessing mediation and interaction effect by each $\mathrm{CpG}$ on the smoking-birthweight association, implemented by the $\mathrm{R}$ 'medflex' package. Second, for genes with multiple significant $\mathrm{CpG}$ sites, we examined their correlations using a heatmap and then, used factor analyses [36] to create a composite score to represent highly correlated CpG sites in a gene, implemented by $\mathrm{R}$ 'psych' package. We then applied the composite score to perform gene-based mediation analysis using the R 'medflex' package. Finally, we estimated a combined mediation effect of the major significant genes, using the structural equation modeling approach [37], implemented by the R 'lavaan' package. The significance was based on $P$ values provided by the VanderWeele-Vansteelandt and the structural equation models, respectively.

\section{Sensitivity analyses}

To further explore if the above mediation estimation differed by fetal sex and maternal race, we performed sexspecific and race-specific mediation analyses to estimate percent mediation by cord DNAm on the smoking-birthweight association, using similar methods as described above. We also tested DNAm $\times$ sex and DNAm $\times$ race (Black vs. non-Black) interactions on birthweight.

\section{Supplementary Information}

The online version contains supplementary material available at https://doi. org/10.1186/s13148-021-01032-6.

Additional file 1: Table 1. Genome-wide DNA methylation association study identified $38 \mathrm{CpG}$ sites significantly associated with maternal smoking during pregnancy in 954 mother-newborn pairs from the Boston Birth Cohort. Additional file 1: Table 2. Genome-wide DNA methylation association study with maternal smoking during pregnancy: Comparison of significant CpGs among total sample, Blacks only, and non-Blacks only. Additional file 1: Table 3. Methylation mediation effect of each of the 38 single $\mathrm{CpG}$ sites on the maternal smoking-newborn birthweight association in 954 mother-newborn pairs from the Boston Birth Cohort. Additional file 1: Table 4. Single CpG, single gene score, and combined multiple gene mediation analysis on the maternal smoking — newborn birthweight association, among 504 male newborns in the Boston Birth Cohort. Additional file 1: Table 5. Single CpG, single gene score, and combined multiple gene mediation analysis on the maternal smokingnewborn birthweight association, among 450 female newborns in the Boston Birth Cohort. Additional file 1: Table 6. Single CpG, single gene score, and combined multiple gene mediation analysis on the maternal smoking-newborn birthweight association, among 679 Black newborns in the Boston Birth Cohort. Additional file 1: Table 7. Single CpG, single gene score, and combined multiple gene mediation analysis on the maternal smoking —newborn birthweight association, among 275 nonBlack newborns in the Boston Birth Cohort. Additional file 1: Table 8. Characteristics of mother-newborn pairs included $(\mathrm{N}=954)$ versus excluded ( $N=7555)$ from this current study. Additional file 1: Figure 1. Illustration of the relationship between maternal smoking categories $(0=$ never, $1=$ quitter, $2=$ current) and GFI1 gene score (Panel A), AHRR cg05575921 (Panel B), and CYP1A1 gene score (Panel C); and between smoking categories ( $0=$ never, $1=$ quitter, $2=$ current) and birthweight (Panel D), in 954 mother-newborn pairs from the Boston Birth Cohort. Additional file 1: Figure 2. Manhattan and Q-Q plots for the EWAS analyses in cord blood in associations with maternal smoking, in Black (Fig 2A) and non-Black subset (Figure 2B). Grey dotted line represents the epigenome-wide significance cut-off after Bonferroni correction, and the red dotted line represents the epigenome-wide significance cut-off with FDR 0.05 ; Genes in red represent known genes associated with smoking as identified in previous studies. Additional file 1: Figure 3. An illustration of our sequential analytical methods to dissect the interplay of maternal smoking $(X)$, cord blood DNA methylation (M: mediator), and newborn birthweight $(Y)$, where $C^{\prime}$ represents the direct effect of $X$ on $Y$. Additional file 1: Figure 4. Distribution of cotinine/hydroxy-cotinine by smoking status: Panel A: cord plasma cotinine/hydroxy-cotinine by maternal smoking status; Panel B: maternal plasma cotinine/hydroxy-cotinine by maternal smoking status. Additional file 1: Figure 5. Flowchart of Study Participants.

\section{Abbreviations}

AHRR: Aryl-hydrocarbon receptors repressor; BBC: Boston Birth Cohort; BMI: Body mass index; CNTNAP2: Contactin associated protein 2; CpG sites: DNA methylation occurs predominantly at locations of cytosines followed by guanine residues (CPG); CYP1A1: Cytochrome P450, family 1, member A1; DoHaD: Developmental origins of health and disease; DNA: Deoxyribonucleic acid; DNAm: DNA methylation; EWAS: Epigenome-wide association study; FDR: False discovery rate; GEAA: Gestational age at delivery; GFI1: Growth factor independent 1 transcriptional repressor; SNP: Single nucleotide polymorphism.

\section{Acknowledgements}

The authors wish to thank the study participants in the BBC, the nursing staff at labor and delivery of the Boston Medical Center, as well as the field team for their contributions to the Boston Birth Cohort.

\section{Authors' contributions}

$R X, X H, H J$, and $X W$ were responsible for study conception. $R X$ was responsible for drafting of the manuscript. XH, GW, LL and XW supervised DNA methylation data generation. XH and BZ performed DNA methylation quality control and data cleaning. RX performed statistical analyses under the guidance and technical support of $\mathrm{XH}, \mathrm{HJ}, \mathrm{BZ}, \mathrm{WH}, \mathrm{TI}$, and $\mathrm{LL}$. XW was responsible for overseeing acquisition of the epidemiological and clinical data as well as biospecimens. All the authors were responsible for critical review and revision of the manuscript and contributed to data interpretations. XH has full access to all the data in the study and takes responsibility for the integrity of the data and the accuracy of the data analysis. All authors read and approved the final manuscript.

\section{Funding}

The Boston Birth Cohort (the parent study) was supported in part by the National Institutes of Health (NIH) grants (R21ES011666, 2R01HD041702, R21HD066471, R01HD086013, R01HD098232, R21Al154233, R01ES031272, R03HD096136, and R01ES031521). This information or content and conclusions are those of the author and should not be construed as the official position or policy of, nor should any endorsements be inferred by the funding agencies. Richard Xu is supported by the Johns Hopkins University Provost's Undergraduate Research Award (PURA). 


\section{Availability of data and materials}

The datasets supporting these findings are not publicly available. Instead, the datasets used and/or analyzed for the current study are available from the corresponding author on reasonable request and after Institutional Review Board review and approval.

\section{Ethics approval and consent to participate}

The study has been approved by the Institutional Review Boards of Boston Medical Center and Johns Hopkins Bloomberg School of Public Health. Written informed consent was obtained from each participant.

\section{Consent for publication}

Not applicable.

\section{Competing interests}

The authors declare that they have no competing interests.

\begin{abstract}
Author details
${ }^{1}$ Department of Computer Science, Whiting School of Engineering, Johns Hopkins University, Baltimore, MD, USA. ${ }^{2}$ Center On the Early Life Origins of Disease, Department of Population, Family and Reproductive Health, Johns Hopkins University Bloomberg School of Public Health, Baltimore, MD, USA. ${ }^{3}$ Department of Biostatistics, Johns Hopkins University Bloomberg School of Public Health, Baltimore, MD 21205, USA. ${ }^{4}$ Department of Civil and Systems Engineering, Whiting School of Engineering, Johns Hopkins University, Baltimore, MD, USA. ${ }^{5}$ Department of Pediatrics, Johns Hopkins University School of Medicine, Baltimore, MD 21205, USA. ${ }^{6}$ Department of Epidemiology, T.H. Chan School of Public Health, Harvard University, Boston, MA, USA. ${ }^{7}$ Department of Biostatistics, T.H. Chan School of Public Health, Harvard University, Boston, MA, USA.
\end{abstract}

Received: 28 September 2020 Accepted: 15 February 2021 Published online: 04 March 2021

\section{References}

1. Tong VT, Dietz PM, Morrow B, D'Angelo DV, Farr SL, Rockhill KM, England $L J$, Centers for Disease Control and Prevention. Trends in smoking before, during, and after pregnancy-Pregnancy Risk Assessment Monitoring System, United States, 40 sites, 2000-2010. MMWR Surveill Summ. 2013;62(6):1-19.

2. Centers for Disease Control and Prevention. Smoking during pregnancy-United States, 1990-2002. MMWR Morb Mortal Wkly Rep. 2004;53(39):911-5.

3. Wang X, Zuckerman B, Pearson C, Kaufman G, Chen C, Wang G, Niu T, Wise $\mathrm{PH}$, Bauchner $\mathrm{H}$, Xu X. Maternal cigarette smoking, metabolic gene polymorphism, and infant birth weight. JAMA. 2002;287(2):195-202.

4. Joubert BR, Felix JF, Yousefi P, Bakulski KM, Just AC, Breton C, Reese SE, Markunas CA, Richmond RC, Xu CJ, et al. DNA methylation in newborns and maternal smoking in pregnancy: genome-wide consortium metaanalysis. Am J Hum Genet. 2016;98(4):680-96.

5. Joubert BR, Haberg SE, Nilsen RM, Wang X, Vollset SE, Murphy SK, Huang Z, Hoyo C, Midttun O, Cupul-Uicab LA, et al. 450K epigenomewide scan identifies differential DNA methylation in newborns related to maternal smoking during pregnancy. Environ Health Perspect. 2012;120(10):1425-31.

6. Zhang B, Hong $X$, Ji H, Tang WY, Kimmel M, Ji Y, Pearson C, Zuckerman B, Surkan PJ, Wang X. Maternal smoking during pregnancy and cord blood DNA methylation: new insight on sex differences and effect modification by maternal folate levels. Epigenetics. 2018;13(5):505-18.

7. Knopik VS. Maternal smoking during pregnancy and child outcomes: real or spurious effect? Dev Neuropsychol. 2009;34(1):1-36.

8. Barker DJ. Fetal origins of coronary heart disease. BMJ. 1995;311(6998):171-4.

9. de Boo HA, Harding JE. The developmental origins of adult disease (Barker) hypothesis. Aust N Z J Obstet Gynaecol. 2006;46(1):4-14.

10. Wang G, Divall S, Radovick S, Paige D, Ning Y, Chen Z, Ji Y, Hong X, Walker SO, Caruso D, et al. Preterm birth and random plasma insulin levels at birth and in early childhood. JAMA. 2014;311(6):587-96.
11. Philips EM, Santos S, Trasande L, Aurrekoetxea JJ, Barros H, von Berg A, Bergstrom A, Bird PK, Brescianini S, Ni Chaoimh C, et al. Changes in parental smoking during pregnancy and risks of adverse birth outcomes and childhood overweight in Europe and North America: an individual participant data meta-analysis of 229,000 singleton births. PLoS Med. 2020;17(8):e1003182.

12. Gluckman PD, Hanson MA, Buklijas T. A conceptual framework for the developmental origins of health and disease. J Dev Orig Health Dis. 2010;1(1):6-18.

13. Hanson M. Understanding the origins of diabetes. JAMA. 2014:311(6):575-6.

14. Feinberg AP. The key role of epigenetics in human disease prevention and mitigation. N Engl J Med. 2018;378(14):1323-34.

15. Starling AP, Liu C, Shen G, Yang IV, Kechris K, Borengasser SJ, Boyle KE, Zhang W, Smith HA, Calafat AM, et al. Prenatal exposure to per- and polyfluoroalkyl substances, umbilical cord blood DNA methylation, and cardio-metabolic indicators in newborns: the healthy start study. Environ Health Perspect. 2020;128(12):127014.

16. Kupers LK, Monnereau C, Sharp GC, Yousefi P, Salas LA, Ghantous A, Page CM, Reese SE, Wilcox AJ, Czamara D, et al. Meta-analysis of epigenomewide association studies in neonates reveals widespread differential DNA methylation associated with birthweight. Nat Commun. 2019;10(1):1893.

17. Agha G, Hajj H, Rifas-Shiman SL, Just AC, Hivert MF, Burris HH, Lin X, Litonjua AA, Oken E, DeMeo DL, et al. Birth weight-for-gestational age is associated with DNA methylation at birth and in childhood. Clin Epigenetics. 2016:8:118

18. Gonzalez-Nahm S, Mendez MA, Benjamin-Neelon SE, Murphy SK, Hogan VK, Rowley DL, Hoyo C. DNA methylation of imprinted genes at birth is associated with child weight status at birth, 1 year, and 3 years. Clin Epigenetics. 2018;10:90

19. Miyake K, Kawaguchi A, Miura R, Kobayashi S, Tran NQV, Kobayashi S, Miyashita C, Araki A, Kubota T, Yamagata Z, et al. Association between DNA methylation in cord blood and maternal smoking: the Hokkaido Study on Environment and Children's Health. Sci Rep. 2018;8(1):5654.

20. Kupers LK, Xu X, Jankipersadsing SA, Vaez A, la Bastide-van GS, Scholtens S, Nolte IM, Richmond RC, Relton CL, Felix JF, et al. DNA methylation mediates the effect of maternal smoking during pregnancy on birthweight of the offspring. Int J Epidemiol. 2015;44(4):1224-37.

21. Witt SH, Frank J, Gilles M, Lang M, Treutlein J, Streit F, Wolf IAC, Peus V, Scharnholz B, Send TS, et al. Impact on birth weight of maternal smoking throughout pregnancy mediated by DNA methylation. BMC Genom. 2018;19(1):290.

22. Relton CL, Davey Smith G. Two-step epigenetic Mendelian randomization: a strategy for establishing the causal role of epigenetic processes in pathways to disease. Int J Epidemiol. 2012;41(1):161-76.

23. Cardenas A, Lutz SM, Everson TM, Perron P, Bouchard L, Hivert MF. Mediation by placental DNA methylation of the association of prenatal maternal smoking and birth weight. Am J Epidemiol. 2019;188(11):1878-86.

24. Hannon E, Schendel D, Ladd-Acosta C, Grove J, Hansen CS, Hougaard DM, Bresnahan M, Mors O, Hollegaard MV, Baekvad-Hansen M, et al. Variable DNA methylation in neonates mediates the association between prenatal smoking and birth weight. Philos Trans R Soc Lond B Biol Sci. 2019:374(1770):20180120.

25. Blum MGB, Valeri L, Francois O, Cadiou S, Siroux V, Lepeule J, Slama R. Challenges raised by mediation analysis in a high-dimension setting. Environ Health Perspect. 2020;128(5):55001.

26. Romanowska J, Haaland OA, Jugessur A, Gjerdevik M, Xu Z, Taylor J, Wilcox AJ, Jonassen I, Lie RT, Gjessing HK. Gene-methylation interactions: discovering region-wise DNA methylation levels that modify SNP-associated disease risk. Clin Epigenetics. 2020;12(1):109.

27. Hong X, Hao K, Ji H, Peng S, Sherwood B, Di Narzo A, Tsai HJ, Liu X, Burd I, Wang G, et al. Genome-wide approach identifies a novel genematernal pre-pregnancy BMI interaction on preterm birth. Nat Commun. 2017:8:15608

28. Aryee MJ, Jaffe AE, Corrada-Bravo H, Ladd-Acosta C, Feinberg AP, Hansen KD, Irizarry RA. Minfi: a flexible and comprehensive Bioconductor package for the analysis of Infinium DNA methylation microarrays. Bioinformatics. 2014;30(10):1363-9.

29. Liu Y, Aryee MJ, Padyukov L, Fallin MD, Hesselberg E, Runarsson A, Reinius $L$, Acevedo N, Taub M, Ronninger M, et al. Epigenome-wide association 
data implicate DNA methylation as an intermediary of genetic risk in rheumatoid arthritis. Nat Biotechnol. 2013;31(2):142-7.

30. Fortin JP, Triche TJ Jr, Hansen KD. Preprocessing, normalization and integration of the Illumina HumanMethylationEPIC array with minfi. Bioinformatics. 2017;33(4):558-60.

31. Ritchie ME, Phipson B, Wu D, Hu Y, Law CW, Shi W, Smyth GK. limma powers differential expression analyses for RNA-sequencing and microarray studies. Nucleic Acids Res. 2015;43(7):e47.

32. Chen J, Behnam E, Huang J, Moffatt MF, Schaid DJ, Liang L, Lin X. Fast and robust adjustment of cell mixtures in epigenome-wide association studies with SmartSVA. BMC Genom. 2017;18(1):413.

33. Bakulski KM, Feinberg Jl, Andrews SV, Yang J, Brown SSLM, Witter F, Walston J, Feinberg AP, Fallin MD. DNA methylation of cord blood cell types: Applications for mixed cell birth studies. Epigenetics. 2016;11(5):354-62.
34. Benjamini Y, Hochberg Y. Controlling the false discovery rate: a practical and powerful approach to multiple testing. J R Stat Soc Ser B (Methodol). 1995:57(1):289-300.

35. VanderWeele TJ, Vansteelandt S. Mediation analysis with multiple mediators. Epidemiol Methods. 2014;2(1):95-115.

36. Revelle W. Hierarchical cluster analysis and the internal structure of tests. Multivar Behav Res. 1979;14(1):57-74.

37. Gunzler D, Chen T, Wu P, Zhang H. Introduction to mediation analysis with structural equation modeling. Shanghai Arch Psych. 2013;25(6):390-4.

\section{Publisher's Note}

Springer Nature remains neutral with regard to jurisdictional claims in published maps and institutional affiliations.
Ready to submit your research? Choose BMC and benefit from:

- fast, convenient online submission

- thorough peer review by experienced researchers in your field

- rapid publication on acceptance

- support for research data, including large and complex data types

- gold Open Access which fosters wider collaboration and increased citations

- maximum visibility for your research: over $100 \mathrm{M}$ website views per year

At BMC, research is always in progress.

Learn more biomedcentral.com/submissions 